\title{
High-Frequency Ultrasonic Dopplerography May be used to Screen for Diabetic Cardiac Autonomic Neuropathy
}

\author{
Zelenina Tatiana ${ }^{1, *}$, Salukhov Vladimir ${ }^{2}$, Volkova Elena $^{3}$, Aleksandr Zemlianoi ${ }^{4}$, and Girina Marina ${ }^{5}$ \\ ${ }^{1}$ Endocrinologist of the Medical Center of the S. M. Kirov Military Medical Academy, St. Petersburg, Russia \\ ${ }^{2}$ Department of Endocrinology, SM Kirov Military Medical Academy, St. Petersburg, Russia \\ ${ }^{3}$ Department of Endocrinology, North-Western State Medical University, St. Petersburg, Russia \\ ${ }^{4}$ Department of Surgery, National Pirogov Medical Surgical Center, Moscow, Russia \\ ${ }^{5}$ Head of the SP Minimax Ltd, St. Petersburg, Russia
}

*Corresponding author: Zelenina Tatiana, SM Kirov Military Medical Academy, Botkinskaya Street 21, 194044, St. Petersburg, Russia, Tel: +79219760986; Fax: +7(812)2923286; E-mail: tzelenina@mail.ru

Received: 30 Sep, 2019 | Accepted: 24 Sep, 2019 | Published: 31 Oct, 2019

Citation: Tatiana Z, Vladimir S, Elena V, Aleksandr Z, Marina G (2019) High-Frequency Ultrasonic Dopplerography May be used to Screen for Diabetic Cardiac Autonomic Neuropathy. Int J Endocrinol Metab Disord 5(2): dx.doi.org/10.16966/2380-548X.160

Copyright: (c) 2019 Tatiana Z, et al. This is an open-access article distributed under the terms of the Creative Commons Attribution License, which permits unrestricted use, distribution, and reproduction in any medium, provided the original author and source are credited.

The new method of high-frequency dopplerography is proposed for early diagnosis of diabetic autonomic neuropathy.

\section{Abstract}

Diabetic neuropathy is the common complication of diabetes and cardiovascular autonomic neuropathy is associated with early morbidity and mortality on diabetic patients. We investigated the potential of High-frequency Ultrasonic Dopplerography for early diagnosis of cardiovascular autonomic neuropathy. 52 diabetic patients with sensorimotor neuropathy were examined. Cardiovascular autonomic neuropathy was detected using several cardiovascular autonomic reflex tests as a gold standard of diagnosis. Microvascular blood flow of finger skin was evaluated at rest as well as in functional tests: with cold impact and occlusion (cuff). The cold test tuned out to be high informative for discrimination of cardiovascular autonomic neuropathy staging and predictive of confirmed/severe stage (sensitivity of marker was $100 \%$, specificity $77 \%$ ). High-frequency Ultrasonic Dopplerography allowed separating of cardiovascular autonomic neuropathy stages. This study is necessary to continue for revealing of all method possibilities.

Keywords: Diabetes; Microcirculation; Microvascular innervations; Cardiovascular autonomic neuropathy

\section{Introduction}

Diabetes Mellitus (DM) is one of the most widespread endocrine pathologies leading to early disability and mortality of patients $[1,2]$. Nervous system impairment occurs on $40-60 \%$ of patients with type 2 DM (T2DM) already at the time of diagnosis [3-6]. Peripheral sensorimotor and autonomic cardiovascular neuropathies (CAN) are the reason for the most severe complications of DM the diabetic foot and painful polyneuropathy [3,7]. According to different researchers the spread of CAN varies significantly depending on the type of diabetes, its duration and complications, age of patients, diagnostic criteria [3,8] and going up to $65 \%$ in patients with T2DM [7]. However, CAN proceeds asymptomatically in most cases or its clinical symptoms are not specific $[9,10]$. Diagnostic criteria and staging of CAN are still being debated. Staging of CAN have the crucial significance because the progressive stages of CAN are associated with increasingly worse prognosis. A complex of cardiovascular autonomic reflex tests known as CARTs or cardiovascular tests of Ewing DJ and Clarke BF are traditionally performed and widely accepted as the gold standard for screening and monitoring CAN $[3,7,11,12]$. However, the combination of CARTs with some additional screening methods may allow more accurate diagnosis of CAN [13,14]. Thus, the search for diagnostic methods to screen CAN in patients with DM is of current interest. Functional abnormalities of the microcirculation have gained significant attention in recent years for their potential pathogenic role in the development of diabetic complications, particularly diabetic neuropathy and diabetic foot problems $[15,16]$.

The most accessible microcirculatory bed is that of the skin, and its functional assessment represents therefore a very tempting approach for the understanding of pathomechanisms that lead to microvascular dysfunction [17]. More recently, Higher-frequency Doppler ultrasound with probes of $20-25 \mathrm{MHz}$ were allowed for the assessment of skin blood flow in healthy subjects and patients with different diseases. The series of clinical studies have demonstrated promising results [18-20]. High-frequency Ultrasonic Dopplerography (HFUD) is commonly used to investigate skin microcirculation in the clinic. It is noninvasive, simple, fast, and economical and effectively identifies peripheral microvascular changes in the wide range of blood vessels [21-24].

The aim of this study was to evaluate the method of HFUD for assessing CAN in patients with $\mathrm{DM}$, to examine its diagnostic 
efficiency in prognosis of CAN compared with traditional modalities (CARTs).

\section{Material and Methods}

\section{Study population}

A pilot prospective study was performed. The 52 patients with T2DM (38 men and 14 women) have been included in the study on the bases of the endocrinology departments of North-Western State Medical University n.a. I. I. Mechnikov and S. M. Kirov Military Medical Academy. All participants gave informed consent to the study which was approved by the Local Ethics Committee. Inclusion criteria were presence of type 2 diabetes, symptoms and/or signs of diabetic sensorimotor neuropathy. Exclusion criteria were poor controlled of diabetes (HbAlc $\geq 10 \%$ ), atrial flutter, fibrillation ore use of pacemaker, severe liver or kidney disease (estimated glomerular filtration rate $(e G F R)<30 \mathrm{ml} / \mathrm{min} / 1.73 \mathrm{~m}^{2}$ ), stage III and IV New York Heart Association (NYHA) heart failure, chronic obstructive pulmonary disease, other causes of peripheral neuropathy, significant peripheral arterial disease defined as Ankle-Brachial Index $\leq 0.9$. No exclusions in relation to current pharmacotherapy were applied. Ten nondiabetic healthy subjects of the same age were recruited for control group of the study.

\section{Data collection and clinical evaluation}

Demographic data were documented including age, sex, height, weight and smoking history. Pertinent medical history (duration of diabetes, history of hypertension, CVD, cerebrovascular disease and microvascular disease (nephropathy and retinopathy) were confirmed from medical records. All measurements have been performed by standard conditions to obtain reliable measurements. Eligible participants were advised to refrain from caffeine, alcohol, smoking and strenuous exercise for 8-10 hours before the study. Demographic data collection and all instrumental manipulations were performed in a temperature controlled room $\left(22-24^{\circ} \mathrm{C}\right)$. After fasting for at least 8 hours, venous blood was collected from participants for measurement of $\mathrm{HbAlc} \%$, serum lipids and serum creatinine on an automated analyzer enzymatically. The Modification of Diet and Renal Disease (MDRD) formula was used to calculate eGFR. All tests were performed after the participants were acclimatized in the examination room and rested for $20 \mathrm{~min}$ in a supine position.

\section{Assessment of microcirculatory blood flow}

Microvascular blood flow of skin at the nail roller of fingers at rest was estimated by method of high frequency ultrasonic dopplerography (HFUD) using the "Minimax-Doppler-K" device (LLC JV "Minimax", Russia, St. Petersburg) [18-20]. Method is based on the Doppler principle: ultrasound is directed towards target tissue, usually the skin. Light is scattered back on red blood cells and is then collected and analyzed by ultrasound probe. The outcome is presented as blood flow or flux which is represented actual linear and volume perfusion values in the vessels of wide range $0.3-2 \mathrm{~mm}$. As every change in blood flow velocity is detected, the signals from blood flow of arterioles represent pulsation as result of left ventricle contraction. Setting the probe (20$25 \mathrm{MHz}$ ) was performed without compression of skin in the field of nail rollers of fingers [20-22].

Microvascular blood flow was registered in the form of patterned Doppler images, which were analyzed after the procedure. Computerbased analysis of Doppler images allowed averaging all velocity parameters registered in the vessel lumen or tissue volume and obtain the weighed mean velocity. The average linear systolic velocity (Vas), average linear diastolic velocity (Vad) and average mean linear blood flow velocity during cardiac cycle (Vam) were derived from weighed mean velocity curve. Blood flow was estimated at rest and during functional tests: cold and occlusive (cuff) ones.

Cold test: It was performed to assess sympathetic adrenergic regulation mechanisms of vessel tone of finger skin. The hand was immersed for one minute in cold water with temperature at $2-4^{\circ} \mathrm{C}$ (floating ice) with the blood flow in the contralateral extremity recorded. The following parameters were recorded automatically: baseline blood flow for three minutes, cold implication with recorded of blood flow for one minute followed by three minutes of post cold exposure blood flow response. Cold test measures used for analysis included maximal decreasing of mean linear blood flow velocity (Vam) on cold impetus and the peak of blood flow in post cold exposure period as a percentage of baseline Vam. These variables were chosen due to their ability to depict the response over time in the context of their previously demonstrated acceptable reliability $[19,23,24]$. In the time of cooling normal blood flow decreased by $30-50 \%$, and after cold exposure it increased by $20-30 \%$ above the initial one. Cold test was considered as the negative (the kept vasoconstriction) in all cases of adequate decrease of blood flow on cold impetus and/or adequate increase after cold exposure. In other cases, the test was regarded as the positive (violation of vascular innervation).

Cuff (occlusive) test: At present the cuff test is regarded as the test used not only for assessing the endothelium function, but also for reflecting state of microcirculatory function in whole [2325]. Since nitrogen oxide is involved in post-occlusive response of microcirculatory blood flow only partially, along with an axonal reflex, calcium-activated potassium channels and other mediators [24-26]. During the test, the manometer cuff, which the pressure was forced on 20-30 $\mathrm{mmHg}$ more than systolic pressure of the patient, was laid on his/her brachium. The compression was performed within 3 minutes, and then the decompression of a brachial artery was quickly obtained. In healthy people after short-term occlusion there was increasing of the skin blood flow by 1.5-3 times in comparison with the baseline. The test was considered as the negative in the cases of blood flow increase in the first or the second minute after short-term occlusion (adequate increase of blood flow). If there was no increase in the blood flow or it was insufficient, test was considered as the positive (pathologically changed reaction of blood flow).

\section{Cardiovascular autonomic reflex tests}

These tests (CARTs) were used as the gold standard clinical testing method and were performed as previously described [27]. All patients underwent a combined investigation of the autonomous regulation of circulation, including: the tilt-table test (the difference in systolic blood pressure change between lying down and passive standing up after 2 minutes), heart rate response to deep breathing (the difference between the maximum and minimum heart rates during each deep expiration and inspiration at 6 breaths per minute), Valsalva maneuver (the ratio of the longest R-R interval shortly after Valsalva maneuver to the shortest R-R interval during Valsalva maneuver), handgrip test (the difference in systolic blood pressure change between baseline and during static exercise), and cold-stress vasoconstriction.

The circulation assessment was performed with the non-invasive beat-to-beat blood pressure monitor Finometer-Pro (FMS, Holland) simultaneous ECG recording. The forearm blood flow was measured by venous occlusion plethysmography using Dohn air-filled plethysmograph.

The scoring system of the CARTs is presented in the table 1. Each normal autonomic functional test was graded as 0 , each borderline 
Table 1: Components and scoring system for the cardiovascular reflex tests.

\begin{tabular}{|l|c|c|c|}
\hline $\begin{array}{c}\text { Cardiovascular autonomic } \\
\text { reflex tests }\end{array}$ & $\begin{array}{c}\text { Normal scores } \\
\text { (values) } \\
\text { (0 points) }\end{array}$ & $\begin{array}{c}\text { Borderline } \\
\text { scores } \\
\text { (0.5 points) }\end{array}$ & $\begin{array}{c}\text { Abnormal } \\
\text { scores } \\
\text { (1 points) }\end{array}$ \\
\hline Valsalva ratio (c.u) & $\geq 1.41$ & $1.40-1.20$ & $\leq 1.19$ \\
\hline Deep breathing test (h.r) & $\geq 15$ & $14-11$ & $\leq 10$ \\
\hline Handgrip test (mm Hg) & $\geq 15$ & $14-11$ & $\leq 10$ \\
\hline Cold-stress vasoconstriction (\%) & $\geq 36$ & $35-25$ & $\leq 24$ \\
\hline Tilt-table test (mm Hg) & $\leq 10$ & $29-11$ & $\geq 30$ \\
\hline
\end{tabular}

test as 0.5 and each abnormal test as 1 . On the basis of the sum of this score, we calculated the total CAN score, which is the sum of the partial scores (minimum 0, maximum 4). According to the Toronto Diabetic Neuropathy Expert Group Recommendation all patients was separated on three groups: CAN 1 (possible/early CAN-one total CAN score was calculated), CAN 2 (definite/confirmed CAN-at least two total CAN scores were found), CAN 3 (severe/advanced $\mathrm{CAN}$-in the cases of orthostatic hypotension in addition to CARTs abnormalities) [3,7].

\section{Assessment of diabetic sensorimotor neuropathy}

The assessment of DPN was based on participants' history and careful physical examination. Symptoms were evaluated by using the Total Symptom Score (TSS) which examines the presence of pain, cramps or aching in the feet. Signs of DPN were evaluated by using Neuropathy Disability Score (NDS). Quantitative sensory testing included the assessment of vibration perception threshold using 128$\mathrm{Hz}$ tuning fork, cutaneous perception (light touch sense) threshold using Semmes-Weinstein monofilaments, and also temperature and pinprick senses. The ankle and knee reflexes were also examined. This clinical scoring system corresponds well with the results of neurophysiological examination and has acceptable sensitivity and specificity for the diagnosis of polyneuropathy when a cut-off point of more than 4 is used [28].

\section{Statistical analysis}

The data were presented as mean \pm Standard Deviation (SD) for continuous variables and as percentages for categorical variables. Chisquare tests were used to compare categorical variables between groups. The differences for continuous variables among the study groups were assessed by Analysis of variance (ANOVA) test. Significance was defined as a $p$ value of less than 0.05 . Statistical procedures were performed with the statistical package STATISTICA v.10.

The sensitivity, specificity, and positive and negative predictive values were calculated separately for each microvascular blood flow tests to evaluate the sensitivity and specificity of method of Highfrequency Ultrasonic Dopplerography in detecting of CAN staging compared with traditional modalities. Sensitivity indicated the truepositive rate and specificity indicated the true-negative rate. Positive predictive value indicated the true-positive results divided by the total positive cases. Negative predictive values indicated the true-negative results divided by the total negative cases.

\section{Results}

\section{Characteristics of the study population}

Average age of patients was $66.2 \pm 7.6$ years old, duration of T2DM was $9.8 \pm 7.0$ years. The symptoms of CAN such as the complaints to feeling of heartbeat at rest, blackout, and dizziness when changing position of a body were revealed only in 10 patients (19\%). Nevertheless CAN was diagnosed for all patients: possible/early CAN (CAN 1) in 31 patients (59\%), definite/confirmed in 9 ones and severe/ advanced in 12 subjects ( 17 and $23 \%$ respectively). The patients with confirmed and severe CAN were merged into the group of CAN 2-3. Initial characteristics of the patients and control group are presented in the table 2.

As shown in the table 2, the participants with different stage of CAN were comparable in age, gender ratio and duration of T2DM, presence of other diabetic microvascular complications, body mass index, and parameters of metabolic control.

Notably, comparisons showed that patients with CAN 2-3 had a more severe sensorimotor neuropathy compared to the subjects with CAN 1. There were significant differences in the presence of ischemic heart disease and arterial hypertension between groups, with those with confirmed/severe CAN much more likely to have macrovascular complications of diabetes than those with early stage.

\section{Comparison of microcirculatory blood flow parameters in the study population}

Initial parameters of microcirculatory blood flow were decreased in all patients with T2DM in comparison with the control group (Table 3). Furthermore significant differences between the groups of the patients with CAN 1 and CAN 2-3 were found according to the HFUD (Table 3 , Graph 1). Thus the average parameters of microvascular blood flow velocity were significantly decreased in the patients with CAN 2-3 in comparing with CAN 1.

\section{Comparison of occlusive (cuff) test results in the study population}

The positive results (pathologically changed reaction of blood flow) of occlusive (cuff) test were revealed for 12 patients (39\%) in the CAN 1 group and for the more than half of patients $(62 \%)$ in the group of CAN 2-3 (Graph 2). The negative tests (adequate increase of blood flow after short-term occlusion) were registered for 19 patients $(61 \%)$ with early CAN and for 8 patients (38\%) with confirmed/severe stages (Figure 1). Only negative results were obtained in the control group.

We analyzed the efficiency of positive occlusive (cuff) test result for diagnosis of confirmed/severe CAN, compared with the results of the standard CARTs. The positive result of occlusive (cuff) test turned out to be most informative for prognosis of abnormal Handgriptest (sensitivity of marker was $40 \%$, specificity of marker was $75 \%$, positive predictive value was $33 \%$, negative predictive value was $80 \%$ ). Moreover, specificity of occlusive (cuff) test for prognosis of confirmed/ severe CAN was $61 \%$, sensitivity was $62 \%$, positive predictive value, and negative predictive value were $52 \%$ and $70 \%$ respectively (Table 4 ).

\section{Comparison of results of cold test in the study population}

The positive results of cold test (pathologically changed microvascular reactivity) were registered in $23 \%$ patients with the early CAN and in all patients (100\%) with confirmed/severe CAN (Figure 1 , Graph 3). Respectively the negative results (adequate decrease of blood flow during application of cold implication and/or an adequate increase of blood flow after cold exposure) were observed in $77 \%$ patients of the CAN 1 group and in no patients with CAN 2. Negative results of cold test were found for all subjects in controls.

The cold test results turned out to be the most informative to predict of cold vasoconstriction (adrenergic vascular innervation) measured by photoplethysmography method. Thus the sensitivity of marker was 
Table 2: Characteristics of the study population.

\begin{tabular}{|c|c|c|c|c|}
\hline \multirow[t]{2}{*}{ Variable } & \multicolumn{2}{|c|}{ Diabetes Individuals } & \multirow[t]{2}{*}{ p-value } & \multirow[t]{2}{*}{ Controls $(n=10)$} \\
\hline & CAN $1(n=31)$ & CAN 2-3 (n=21) & & \\
\hline Age, years & $70.0 \pm 9.8$ & $65.7 \pm 7.0$ & 0.26 & $55.8 \pm 7.9$ \\
\hline Gender (m/f) & $22 / 9$ & $16 / 5$ & 0.94 & 5-May \\
\hline Duration of T2DM, years & $9.9 \pm 7.3$ & $9.2 \pm 5.4$ & 0.87 & - \\
\hline Diabetic retinopathy (\%) & $13(42 \%)$ & $10(48 \%)$ & 0.68 & - \\
\hline Diabetic nephropathy (\%) & $11(36 \%)$ & $8(38 \%)$ & 0.85 & - \\
\hline $\begin{array}{l}\text { Duration of diabetic sensorimotor neuropathy } \\
\text { (years) }\end{array}$ & $2.4 \pm 2.1$ & $2.0 \pm 1.9$ & 0.76 & - \\
\hline $\begin{array}{l}\text { Severity of sensorimotor neuropathy-NDS } \\
\text { (points) }\end{array}$ & $8.4 \pm 2.9$ & $11.2 \pm 3.2$ & 0.06 & - \\
\hline Ischemicheartdisease (\%) & $20(65 \%)$ & $20(95 \%)$ & 0.01 & - \\
\hline Arterial hypertension (\%) & $28(90 \%)^{*}$ & $21(100 \%)^{*}$ & 0.14 & $3(30 \%)$ \\
\hline BMI $\left(\mathrm{kg} / \mathrm{m}^{2}\right)$ & $31.4 \pm 5.7$ & $31.3 \pm 5.7$ & 0.95 & $28.4 \pm 2.4$ \\
\hline $\mathrm{HbA1c}(\%)$ & $7.0 \pm 1.2^{*}$ & $7.2 \pm 0.9 *$ & 0.27 & $5.8 \pm 1.7$ \\
\hline tChs (mmol/l) & $4.3 \pm 1.2$ & $4.2 \pm 1.1$ & 0.77 & $5.0 \pm 1.2$ \\
\hline Ch-LDL (mmol/l) & $1.9 \pm 0.8$ & $2.5 \pm 1.0$ & 0.16 & $2.9 \pm 1.1$ \\
\hline Ch-HDL (mmol/l) & $1.3 \pm 0.2$ & $1.1 \pm 0.4$ & 0.6 & $1.0 \pm 0.5$ \\
\hline TG (mmol/l) & $1.4 \pm 0.7$ & $1.5 \pm 1.0$ & 0.88 & $1.9 \pm 1.5$ \\
\hline \multicolumn{5}{|l|}{ Cardiovascular autonomic reflex tests } \\
\hline Valsalva ratio (c.u) & $1.5 \pm 0.5$ & $1.2 \pm 0.2^{*}$ & 0.03 & $1.48 \pm 0.2$ \\
\hline Handgrip test (mm Hg) & $18.3 \pm 3.6$ & $9.4 \pm 6.7^{*}$ & 0.02 & $18.5 \pm 6.6$ \\
\hline Cold-stress vasoconstriction (\%) & $31.2 \pm 9.7^{*}$ & $17.3 \pm 10.6^{*}$ & 0.04 & $39.6 \pm 12.0$ \\
\hline Tilt-table test (mm Hg) & $6.3 \pm 6.1$ & $20.7 \pm 13.5^{*}$ & 0.00002 & $5.9 \pm 8.0$ \\
\hline Deep breathing test (h.r) & $11.6 \pm 2.0^{*}$ & $9.4 \pm 2.0^{*}$ & 0.04 & $18.7 \pm 2.6$ \\
\hline
\end{tabular}

${ }^{*} \mathrm{p}<0.05$ in comparison with control group

T2DM-type 2 Diabetes Mellitus; NDS-scale of Neuropathic Disability Score; BMI-Body Mass Index; HbA1c\%-Glycated Hemoglobin; tChs-Total Cholesterol; Ch-LDL-Cholesterol of Low Density Lipoprotein; Ch-HDL- Cholesterol of High Density Lipoprotein; TG-Triglycerides

Table 3: Comparison of the parameters of skin microcirculatory blood flow in the study population.

\begin{tabular}{|c|c|c|c|c|}
\hline \multirow{2}{*}{ Variable } & \multicolumn{2}{|c|}{ Diabetes individuals $(\mathbf{n = 5 2 )}$} & p-level & Controls $(\mathbf{n = 1 0})$ \\
\cline { 2 - 5 } & CAN1 $(\mathrm{n}=31)$ & CAN2-3(n=21) & & \\
\hline $\operatorname{Vas}(\mathrm{cm} / \mathrm{sec})$ & $6.2 \pm 4.2^{*}$ & $3.8 \pm 3.7^{*}$ & 0.003 & $7.9 \pm 0.6$ \\
\hline $\operatorname{Vad}(\mathrm{cm} / \mathrm{sec})$ & $2.8 \pm 2.6^{*}$ & $1.8 \pm 2.3^{*}$ & 0.018 & $4.2 \pm 0.5$ \\
\hline $\operatorname{Vam}(\mathrm{cm} / \mathrm{sec})$ & $3.9 \pm 3.1^{*}$ & $2.5 \pm 2.7^{*}$ & 0.005 & $6.7 \pm 0.5$ \\
\hline
\end{tabular}

$*_{p}<0.05$ in comparison with control group

Vas-average linear systolic velocity; Vad-average linear diastolic velocity; Vam-average mean linear blood flow velocity during cardiac cycle

Table 4: Diagnostic efficiency of functional tests for prognosis of confirmed/severe cardiac autonomic neuropathy in the patients with type 2 diabetes and sensorimotor neuropathy.

\begin{tabular}{|l|c|c|c|c|}
\hline \multicolumn{1}{|c|}{ Tests } & Sensitivity,\% & Specificity,\% & +PV,\% & -PV,\% \\
\hline Cold-test & 100 & 77 & 75 & 100 \\
\hline Occlusive (cuff) test & 62 & 61 & 52 & 70 \\
\hline
\end{tabular}

+PV: Positive predictive value; -PV: Negative predictive value
$93 \%$, specificity of marker was $80 \%$, positive predictive value was $93 \%$, and negative predictive value was $80 \%$.

The cold test was also the most informative for discrimination of CAN staging and predictive of confirmed/severe CAN and tolerance to orthostasis (sensitivity of marker was $100 \%$, specificity of marker was $77 \%$, positive predictive value was $75 \%$, negative predictive value was $100 \%$ ) (Table 4 ).

Thus the positive cold test result demonstrated high sensitivity, but relatively low specificity for prognosis of advanced stages of CAN. This finding emphasize that in the cases of positive cold test result the risk of confirmed/severe CAN is sufficiently high, but not obligatory. However in the cases of the negative test (kept vasoconstriction), there is a strong evidence to suggest that the patients has no severe autonomic dysfunction.

Thus we suggested performing especially cold test for diagnosis of confirmed/severe CAN in patients with diabetic sensorimotor neuropathy.

\section{Discussion and Conclusions}

CAN, widespread microvascular complication of DM, occurs, according to different authors, among $20-50 \%$ of the patients that depends on methods of diagnosis $[4,6,9,10]$. CAN is difficult to 


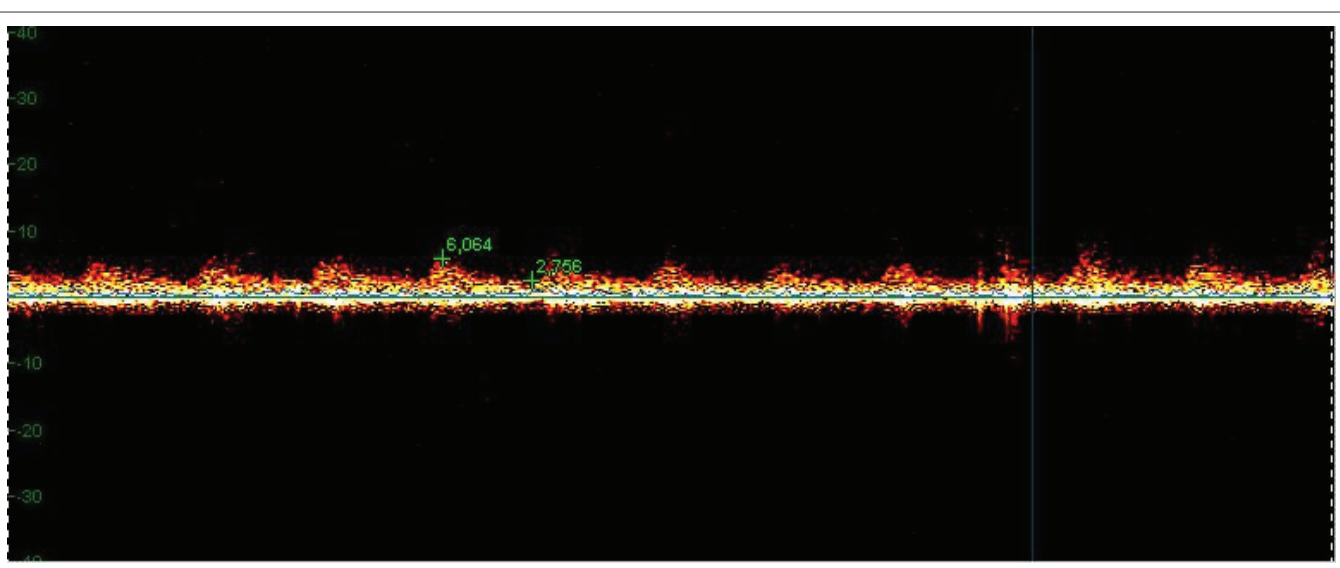

Graphic 1: The patient A. Microcirculatory blood flow of skin at the baseline. Its parameters are in the normal range.

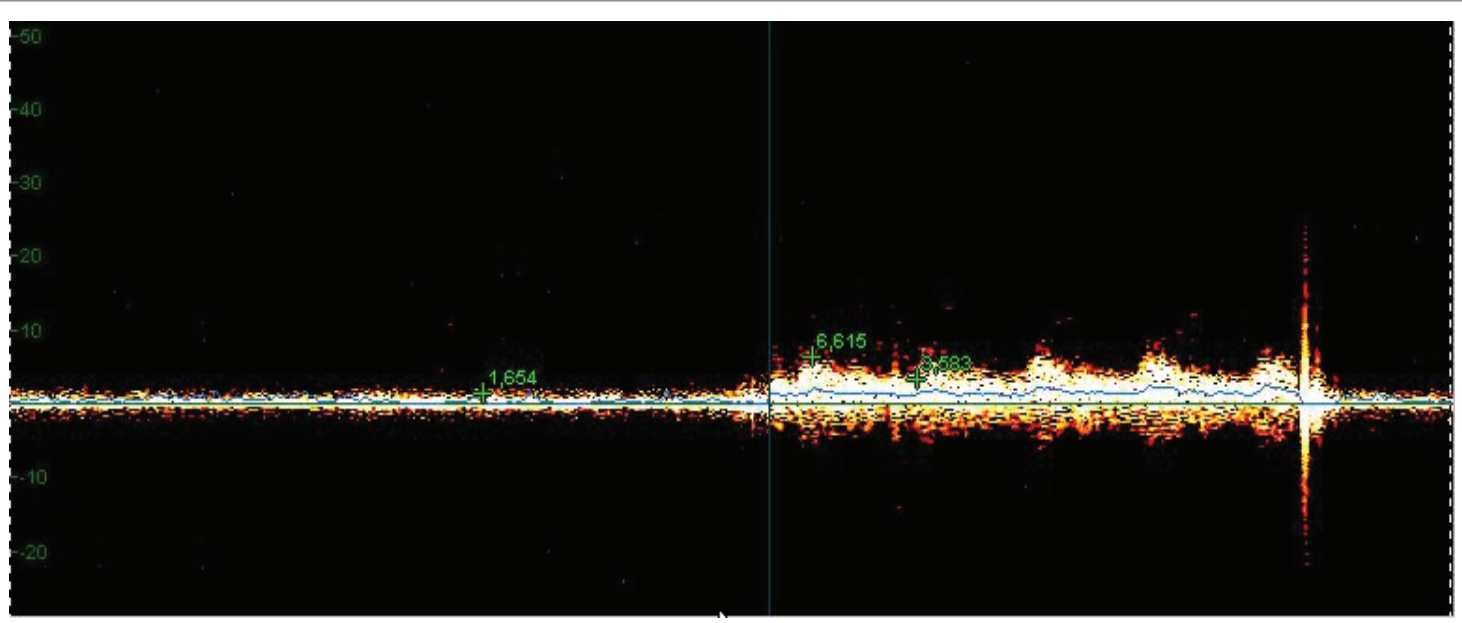

Graphic 2: The patient A. Reaction of the microcirculatory blood flow on occlusive (cuff) test. Nonadequate increasing of microcirculatory blood flow on occlusive (cuff) test. Positive (pathologically changed) reaction of blood flow.

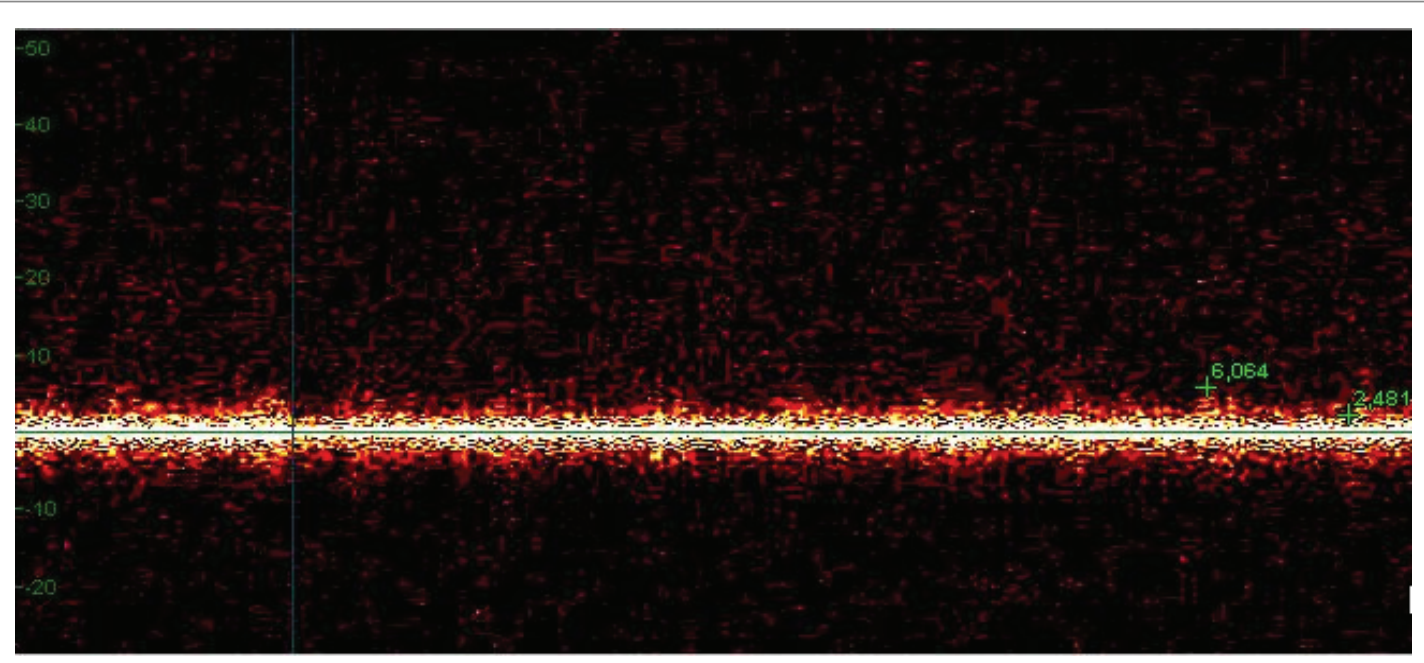

Graphic 3: The patient A. There is no decreasing of microcirculatory blood flow on cold stimulus. Positive (pathologically changed) reaction of blood flow. 


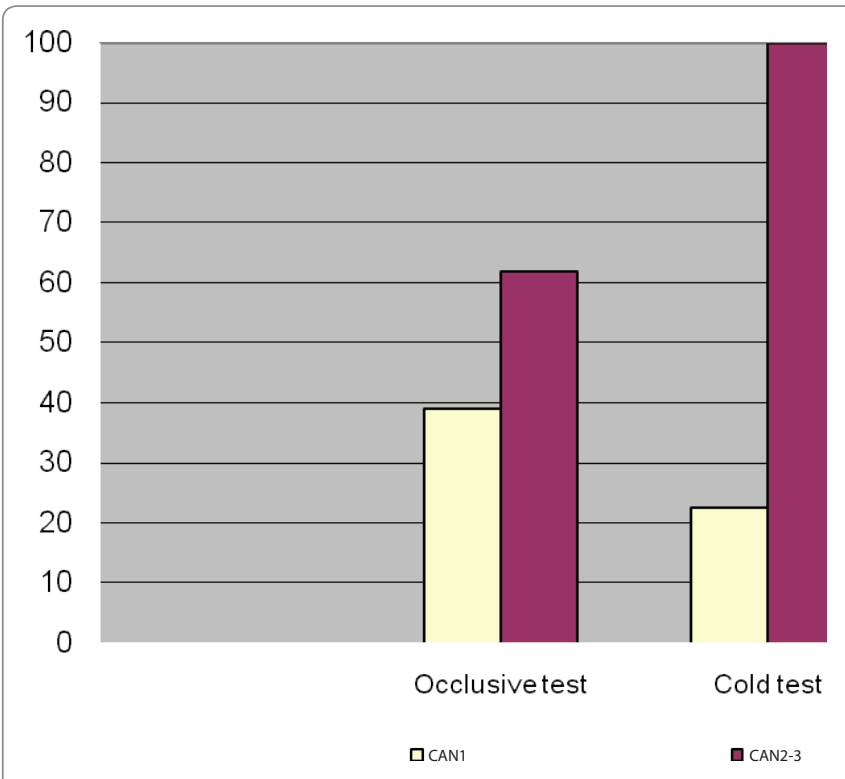

Figure 1: The percent of the patients with positive results of occlusive and cold tests among the CAN 1 and CAN 2-3 study groups.

diagnose at onset. Many diabetic patients remain asymptomatic during the long incubation period, and symptoms when they occur are often not related to damage severity. The groups of high risk CAN patients include all patients with other microvascular complications of DM, particularly with sensorimotor neuropathy. Selection of early and confirmed/severe CAN has crucial significance. Since functional changes are reversible and respond to therapeutic treatment whereas the patients with confirmed, especially severe neuropathy have a high risk of severe complications and are required careful medical observation $[4,6,9,10]$. Thus the present study provides the number of important observation.

First, our data confirms the fact that all of diabetic patients with sensorimotor neuropathy are suffered from CAN. Moreover the definite/confirmed and severe/advanced stages of CAN are diagnosed more than in $40 \%$ subjects. However clinical symptoms of this diabetic complication are presented in less than $20 \%$ cases.

Secondly, present data failed to show any differences in demographic and metabolic parameters between patients with early or definite/ confirmed CAN. This further emphasizes the previous findings that number of parameters could not be suggested to determine the CAN stages $[3,4,6]$. Nevertheless the severe sensorimotor neuropathy and presence of diabetic macrovascular complications such as arterial hypertension and ischemic heart disease may be helpful for prognosis of confirmed/severe CAN.

Thirdly, most important findings were obtained regarding CAN and microvascular impairments. Although the definitive pathophysiology of CAN remains elusive, accumulating evidence indicates the source of a variety of diabetic complications is microvascular lesion. Microvascular dysfunction (pathology) is central to the development of neuropathy. In addition, both sensory and autonomic neuropathies are proposed to contribute to a reduction in functional capacity of the microvasculature $[15,16,29]$. The increasing evidence of the contribution of microvascular dysfunction to neurological complications supports a role for assessments microvascular function in clinical practice to assist in determining these patients at risk.
The recent development of noninvasive techniques such as HFUD, that can reliably quantity blood flow in the skin microcirculation, has made it possible to study changes in microvascular function in patients with diabetes [18-20].

Notably some studies provide evidences that resting dermal microvascular perfusion is apparently not affected in diabetes and the functionality of the microvessels may be a more sensitive indicator of complications $[17,30]$.

In the contrast with these data our findings shows decreasing of baseline blood flow in diabetic patients progressively with the CAN stages. Thus with each stage of CAN with higher number the Vam of microvascular blood flow were lower. The main reason that may explain this discrepancy is including only patients with sensorimotor neuropathy in the present study. Previous studies demonstrated that microvascular diminished progressively from patients with prediabetes to diabetic sensorimotor neuropathy and foot complications $[15,16]$.

The method for the assessment of microvascular function and the site of measurement vary largely according to the aspect of microvascular regulation to be investigated and different stimuli are used to determine microvascular response/reactivity $[17,30]$.

Thus the icy water immersion of the contralateral arm (the cold trial) tests influence of peripheral or autonomic neuropathy (sympatheticdriven vasoconstriction). Dual sympathetic innervation of the skin (vasoconstrictor as well as vasodilator nerves) was first demonstrated in the 1930s. The cold immersion of the contralateral arm causes reflex activation of sympathetic vasoconstrictor nerves, resulting in cutaneous vasoconstriction and decreases in skin blood flow of examined hand. Furthermore sympathetic active vasodilator nerves activated after cold exposure are responsible for most of substantial vasodilation that occur $[15,17,30]$.

The present data suggest good diagnostic efficiency of results of cold test for discrimination of definite/confirmed CAN among diabetic patients with sensorimotor neuropathy. Thus the high sensitivity of the method (100\%) allows excluding the subjects with early CAN in cases of positive result of probe. It can be explained by the fact that in cases of confirmed/severe CAN there are always impairment of peripheral vascular innervation and without this impairment severe CAN does not develop.

The following test-occlusive (cuff) one in general reflected microvascular regulation as function of endothelium, neurovascular function and influence of autonomic neuropathy [23-26]. Notably in the present study this method demonstrate lower diagnostic efficiency for prognosis of definite/confirmed CAN in compared with cold test (specificity and sensitivity $66 \%$ and $61 \%$ respectively). This evidence emphasizes the fact that occlusive (cuff) test results depend on different factors and couldn't be suggested for determination of CAN.

Thus we proposed to perform cold test as high informative for discrimination of CAN staging and selection of the early CAN (100\% specificity) in the cases of negative tests result and diagnosis of the severe CAN for patients with its positive results (sensitivity 77\%).

There were some limitations in our study. This study was a crosssectional study and the sample size was small. In addition, long-term follow-up observation is lacking. A prospective study with a large sample size including patients not only with sensorimotor neuropathy other groups of patients (diabetic patients without sensorimotor polyneuropathy, patients with prediabetes, metabolic syndrome, obesity) is needed to investigate an association between the HFUD 
microvascular blood flow parameters and CAN progression, and confirm the advantages of the method for CAN screening.

Our study therefore does not explain the exact mechanisms underlying the reactivity. There are several external and internal factors which may influence the results. This includes measurement technique and pharmacological agents including antihypertensives and drugs for dyslipidemia or insulin which were not excluded from this study as they are in widespread use in the target populations.

There are a range of variables that may be assessed within the functional test measurement. In order to limit the number of variables in the analysis we combined several parameters as qualitative variable (positive or negative test result). It is possible that other variables (area under the curve during and post test application, magnitude of the peak, time to maximum decreasing or increasing) may provide additional information relating microvascular function and CAN.

However it does not affect the validity of the conclusions regarding the high diagnostic efficiency of cold test by the method of HFUD for discrimination of CAN and prognosis definite/confirmed CAN in diabetic patients with sensorimotor neuropathy.

Summary, our findings suggest that functional test with cold implication provides additional information about presence and stage of CAN in diabetic patients with sensorimotor neuropathy and may be helpful for screening of CAN.

\section{Acknowledgements}

The cooperation of the patients and many staff at the endocrinology departments is much appreciated. The authors would like to thank Mamontov O. V. (Almazov National Medical Research Centre, St. Petersburg, Russia) for his methodology help and assistance in the performing of CARTs. This research did not receive any specific grant from funding agencies in the public, commercial or not-for-profit sectors.

\section{Disclosure}

All authors read and approved the final manuscript.

\section{Competing Interests}

The authors declare no conflict of interests.

\section{References}

1. Brownlee M (2005) The pathobiology of diabetic complications: a unifying mechanism. Diabetes 54: 1615-1625.

2. Valensi P, Pariès J, Attali JR, French Group for Research and Study of Diabetic Neuropathy (2003) Cardiac autonomic neuropathy in diabetic patients: influence of diabetes duration, obesity, and microangiopathic complications--the French multicenter study. Metabolism 52: 815-820.

3. Tesfaye S, Boulton AJ, Dyck PJ, Freeman R, Horowitz M, et al. (2010) Diabetic neuropathies: update on definitions, diagnostic criteria, estimation of severity, and treatments. Diabetes Care 33: 22852293.

4. Vinik Al, Maser RE, Mitchell BD, Freeman R (2003) Diabetic autonomic neuropathy. Diabetes Care 26: 1553-1579.

5. Boulton AJ, Vinik Al, Arezzo JC, Bril V, Feldman EL, et al. (2005) Diabetic neuropathies: a statement by the American Diabetes Association. Diabetes Care 28: 956-962.

6. Ziegler D (2001) Diagnosis and treatment of diabetic autonomic neuropathy. Curr Diab Rep 1: 216-227.
7. Spallone V, Ziegler D, Freeman R, Bernardi L, Frontoni S, et al. (2011) Cardiovascular autonomic neuropathy in diabetes: clinical impact, assessment, diagnosis and management. Diabetes Metab Res Rev 27: 639-653.

8. Low PA, Benrud-Larson LM, Sletten DM, Opfer-Gehrking TL, Weigand SD, et al. (2004) Autonomic symptoms and diabetic neuropathy: a population-based study. Diabetes Care 27: 2942-2947.

9. Kempler P, Tesfaye S, Chaturvedi N, Stevens LK, Webb DJ, et al. (2002) Autonomic neuropathy is associated with increased cardiovascular risk factors: the EURODIAB IDDM Complications Study. Diabet Med 19: 900-909.

10. Wheeler SG, Ahroni JH, Boyko EJ (2002) Prospective study of autonomic neuropathy as a predictor of mortality in patients with diabetes. Diabetes Res Clin Pract 58: 131-138.

11. Ewing DJ, Clarke BF (1982) Diagnosis and management of diabetic autonomic neuropathy. Br Med J (Clin Res Ed) 285: 916-918.

12. Lin K, Wei L, Huang Z, Zeng Q (2017) Combination of Ewing test, heart rate variability, and heart rate turbulence analysis for early diagnosis of diabetic cardiac autonomic neuropathy. Medicine (Baltimore) 96: e8296.

13. He T, Wang C, Zuo A, Liu P, Zhao R, et al. (2017) Electrochemical Skin Conductance May Be Used to Screen for Diabetic Cardiac Autonomic Neuropathy in a Chinese Population with Diabetes. J Diabetes Res 2017: 8289740 .

14. Casellini CM, Parson HK, Richardson MS, Nevoret ML, Vinik Al (2013) Sudoscan, a noninvasive tool for detecting diabetic small fiber neuropathy and autonomic dysfunction. Diabetes Technol Ther 15: 948-953.

15. Malik RA, Tesfaye S, Thompson SD, Veves A, Sharma AK, et al. (1993) Endoneurial localization of microvascular damage in human diabetic neuropathy. Diabetologia 36: 454-459.

16. Lanting SM, Barwick AL, Twigg SM, Johnson NA, Baker MK, et al. (2017) Post-occlusive reactive hyperaemia of skin microvasculature and foot complications in type 2 diabetes. J Diabetes Complications 31: $1305-1310$

17. Stirban A (2014) Microvascular dysfunction in the context of diabetic neuropathy. Curr Diab Rep 14: 541.

18. Lenasi H, Potočnik N, Petrishchev N, Papp M, Egorkina A, et al. (2017) The measurement of cutaneous blood flow in healthy volunteers subjected to physical exercise with ultrasound Doppler imaging and laser Doppler flowmetry. Clin Hemorheol Microcirc 65: 373-381.

19. Zelenina T, Zemlyanoy A, Vorokhobina N (2017) Diagnosis of diabetic cardiac autonomic neuropathy by high-frequency ultrasonic dopplerography. NEURODIAB, Portugal.

20. Lee $\mathrm{CH}$, Kim E, Kim D (2018) Detection of atrial fibrillation during pulpal blood flow assessment using Doppler ultrasound: a case report. Dentomaxillofac Radiol 47: 20170354.

21. White DN (1982) Johann Christian Doppler and his effect--a brief history. Ultrasound Med Biol 8: 583-591.

22. Kang BJ, Park J, Kim J, Kim HH, Lee C, et al. (2015) High-frequency dual mode pulsed wave Doppler imaging for monitoring the functional regeneration of adult zebrafish hearts. J R Soc Interface 12: 20141154.

23. Roustit M, Cracowski JL (2013) Assessment of endothelial and neurovascular function in human skin microcirculation. Trends Pharmacol Sci 34: 373-384

24. Roustit M, Cracowski JL (2012) Non-invasive assessment of skin microvascular function in humans: an insight into methods. Microcirculation 19: 47-64. 
25. Schuler D, Sansone R, Freudenberger T, Rodriguez-Mateos A, Weber G, et al. (2014) Measurement of endothelium-dependent vasodilation in mice--brief report. Arterioscler Thromb Vasc Biol 34: 2651-2657.

26. De Boer MP, Meijer RI, Wijnstok NJ, Jonk AM, Houben AJ, et al. (2012) Microvascular dysfunction: a potential mechanism in the pathogenesis of obesity-associated insulin resistance and hypertension. Microcirculation 19: 5-18.

27. Mamontov OV, Babayan L, Amelin AV, Giniatullin R, Kamshilin AA (2016) Autonomous control of cardiovascular reactivity in patients with episodic and chronic forms of migraine. J Headache Pain 17: 52.
28. Valk GD, de Sonnaville JJ, van Houtum WH, Heine RJ, van Eijk JT, et al. (1997) The assessment of diabetic polyneuropathy in daily clinical practice: reproducibility and validity of Semmes Weinstein monofilaments examination and clinical neurological examination. Muscle Nerve 20: 116-118.

29. Eleftheriadou I, Tentolouris A, Grigoropoulou P, Tsilingiris D, Anastasiou I, et al. (2019) The association of diabetic microvascular and macrovascular disease with cutaneous circulation in patients with type 2 diabetes mellitus. J Diabetes Complications 33: 165-170.

30. Charkoudian N (2010) Mechanisms and modifiers of reflex induced cutaneous vasodilation and vasoconstriction in humans. J Appl Physiol 109: 1221-1228. 